\title{
Current status and future perspectives of laparoscopic radical surgery for advanced gastric cancer
}

\author{
Takahiro Kinoshita, Akio Kaito \\ Gastric Surgery Division, National Cancer Center Hospital East, Chiba, Japan \\ Contributions: (I) Conception and design: T Kinoshita; (II) Administrative support: None; (III) Provision of study materials or patients: T Kinoshita; \\ (IV) Collection and assembly of data: T Kinoshita; (V) Data analysis and interpretation: All authors; (VI) Manuscript writing: All authors; (VII) Final \\ approval of manuscript: All authors. \\ Correspondence to: Takahiro Kinoshita, MD, PhD, FACS. Gastric Surgery Division, National Cancer Center Hospital East, 6-5-1 Kashiwanoha, \\ Kashiwa, 277-8577 Chiba, Japan. Email: takkinos@east.ncc.go.jp.
}

\begin{abstract}
Laparoscopic radical gastrectomy with lymph node dissection has widely penetrated to East Asian countries, where incidence of gastric cancer occurrence is higher than the rest of the world. Laparoscopic distal gastrectomy for cStageI disease is regarded as one of the option in daily practice in the latest Japanese guidelines; however its applicability to more advanced disease (Stage II/III) is still under debate. Actually, operative techniques of laparoscopic D2 dissection is being matured, but still, necessity of total omentectomy, splenic hilar dissection, management of bulky nodes or large primary tumor, high-level anastomosis in esophageal invasion cases, and extensive peritoneal lavage can be raised as technical limitations. In the future perspective, further technological innovation, including next generation surgical robot, may help surgeons overcome these difficulties. Currently, three large-scaled randomized phase-III clinical trials are ongoing in East Asia, in which patients' accruals have been already completed. With coming long-term outcomes of these well-designed studies, oncological validity of laparoscopic approach will be adequately elucidated.
\end{abstract}

Keywords: Laparoscopic surgery (LS), advanced gastric cancer (AGC), randomized clinical trial (RCT)

Received: 28 March 2017; Accepted: 07 April 2017; Published: 09 May 2017.

doi: $10.21037 / \operatorname{tgh} .2017 .04 .05$

View this article at: http://dx.doi.org/10.21037/tgh.2017.04.05

\section{Introduction}

Laparoscopic radical gastrectomy with lymph node dissection has widely penetrated to East Asian countries, where incidence of gastric cancer occurrence is higher than the rest of the world. In the latest guidelines (English $4^{\text {th }}$ version) published by the Japanese Gastric Cancer Association (1), laparoscopic distal gastrectomy for cStage I disease is regarded as one of the options in daily clinical practice. So far, several valuable evidences have been published to prove acceptable short-term as well as longterm outcomes regarding applicability of laparoscopic distal gastrectomy for cStageI cancer. On the other hand, from technical or oncological viewpoint, feasibility and safety of laparoscopic surgery (LS) to more advanced gastric cancer (AGC) (cStageII/III) is still under debate. A golden standard of surgical treatment for AGC is R0 resection combined with D2 lymph node dissection, which is now recognized worldwide. Actually, technically laparoscopic radical gastrectomy with D2 dissection is being matured; however, still technical limitation can be raised and persuasive scientific evidences are lacking to support this applicability at present.

\section{Technical difficulties or limitation for application of LS to AGC}

\section{Total omentectomy}

In the latest Japanese guidelines, it is mentioned that total omentectomy combined with D2 lymph node dissection is recommended in the treatment of patients with T3 or 
T4 infiltration (1). Furthermore, historically, bursectomy, which removes the whole sac of the bursa omentalis, including the anterior leaf of transverse colon mesentery as well as the anterior capsule of pancreas body for prophylactic local control in addition to total omentectomy, had been performed by some experienced surgeons in Japan (2). The theoretical rationale for bursectomy is to reduce the risk of peritoneal recurrences by removing the peritoneum which might contain micrometastases. Bursectomy had been thought to be effective for tumors with serosal exposure positive cases, especially for those located on the posterior wall. Recently, the primary result of JCOG1001 (UMIN000003688) has been published in ASCO 2017 meeting (3), which demonstrated no survival difference between omentectomy versus bursectomy for T3/T4 tumors diagnoses with surgical findings, though bursectomy was not related with increased morbidity or mortality. Thus, now bursectomy is not recommended as a standard procedure for AGC in Japan. Performing complete bursectomy seemed a huge hurdle for LS; in contrast total omentectomy seems not impossible for experienced surgeons. Beyond this discussion, currently there is a clinical question, whether even omentectomy is really necessary for all of the AGC. If partial omentectomy is enough for some population of AGC, the hurdle for LS will be further reduced. However, several researchers insisted that in some metastatic nodes extra-nodal expansion is recognized, which means cancer cell spread out of lymph node capsule to the adjacent adipose tissue $(4,5)$. This might be one of the rationales for performing omentectomy in AGC. It is also well known that extra-nodal expansion is a poor prognostic factor (4), thus further investigation for this phenomenon should be conducted to conclude whether routine omentectomy is necessary or not.

\section{Splenic bilar dissection for proximal gastric cancer}

For proximal advanced cancer, lymph node dissection around the splenic hilar region has been thought to be indispensable. In Japanese guidelines, No. 10 nodal station has been long time included within the extent of D2 dissection in the treatment of proximal AGC (1). From historical aspect, splenectomy has been employed as a tool to completely remove No. 10 and No. 11d lymph node stations, even as a prophylactic intent in Japan. In 2016, the final result of JCOG 0110 (UMINC000000004) has been disclosed (6), which compared splenectomy versus non-splenectomy for proximal AGC not invading the greater curvature line. Finally, there was no difference in long-term survival rate; moreover splenectomy group was associated with increased incidence of morbidity. Therefore, splenectomy is not recommended as a standard treatment now, unless tumors involve the greater curvature line. Following these results, nowadays, there is a consensus in Japan that complete lymph node dissection of No. 10/11d is not required for most of proximal AGC. If so, LS may be an ideal intervention for performing such procedures, because there is no need to struggle with complicated anatomy. Even if splenic hilar dissection is required for tumors invading the greater curvature line, in principle, laparoscopic approach has a potential advantage for maneuvers in deep operative fields like the splenic hilum. Additionally recent progress of three-dimensional computed tomography provides us helpful images of anatomical reconstruction of the splenic hilum, which has a marked anatomical diversity depending on individuals (7). However, for some tough cases, such as invading the splenogastric ligament or the pancreas tail, applicability or safety of laparoscopic maneuver seems still questionable.

\section{Bulky positive nodes or large primary tumor}

Probably, majority of surgeons may agree that laparoscopic procedure has limitation to manage tumors with bulky metastasis-positive nodes or large primary tumors (type 3 tumors $>8 \mathrm{~cm}$ or type 4 ), with concerns of spillage of cancer cells by manipulation with endoscopic forceps. Especially, loss of tactile sense seems to be an important limitation of laparoscopic procedures compared to open surgery. For such cases, currently effectiveness of neoadjuvant chemotherapy using powerful modern regimen is under investigation by several prospective clinical studies. LS for patients treated by neoadjuvant chemotherapy in advance is also technically demanding, because after such treatment unusual tissue fibrosis or edema sometimes exists, which may further increase surgical difficulty.

\section{Esophageal invasion}

This situation is also significantly increase surgical difficulty. Visibility around the lower mediastinal space via the esophageal hiatus using laparoscopic magnified view seems preferable for dissection. Actually, some researchers emphasized the feasibility and effectiveness of laparoscopic procedures for tumors with gastric cancer with esophageal invasion or adenocarcinoma at the esophagogastric junction 
Table 1 Current ongoing large-scaled randomized clinical studies comparing laparoscopic versus open distal gastrectomy for advanced gastric cancer conducted in East Asian countries

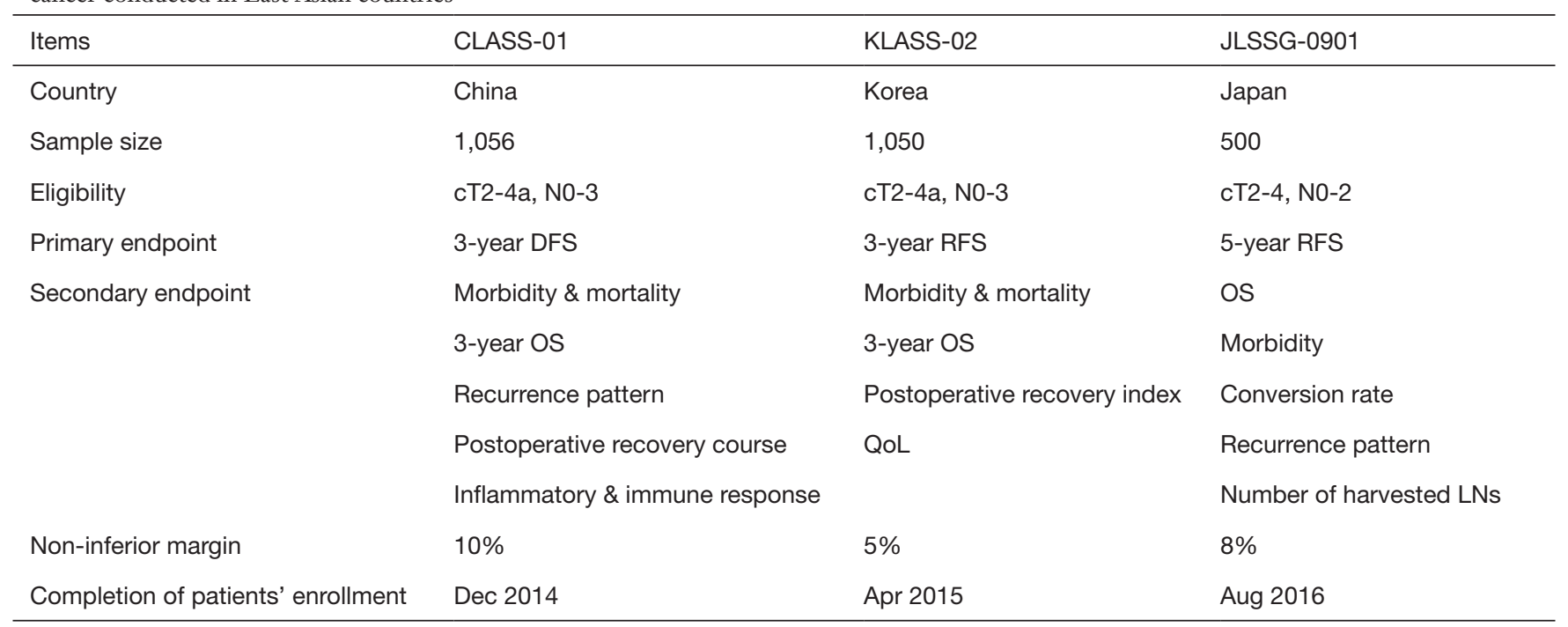

DFS, disease free survival; RFS, relapse free survival; OS, overall survival; QoL, quality of life; LN, lymph node.

(8-10). However, still, intracorporeal anastomosis at the high-level in the lower mediastinum seems challenging even for skilled surgeons. In our institutions, for such cases, linear-stapled esophagojejunal anastomosis, overlap method (11), is basically selected, with widening the esophageal hiatus by division of the diaphragmatic crus. Compared to circular-stapled method, linear-stapled method provides much better visibility during the anastomosis due to smaller shaft of the device. Presently, such procedures should be performed only by well-experienced surgical team.

\section{Peritoneal lavage}

Some investigator emphasized the impact of extensive intraoperative peritoneal lavage using a large amount of saline solution for preventing peritoneal recurrences, which theological basis is to reduce free cancer cell in the peritoneal cavity. Actually, some prospective clinical studies are ongoing to evaluate the survival impact of this prophylactic simple treatment. LS can provide an excellent magnified view, but such a gross procedure seems to be a weak point. Sending a large amount of saline solution is not difficult, but shaking the intestines well in the peritoneal cavity or complete evacuation of the solution using current available laparoscopic device seems more difficult compared to open surgery. If positive impact of extensive peritoneal lavage is verified by the ongoing trials, some effective laparoscopic measures should be developed.

\section{Previous studies and current ongoing multi- institutional large-scaled prospective studies}

So far most of available data regarding long-term outcomes are retrospective clinical studies with from small-sized to relative large-sized samples from both western and eastern countries. Majority of them reported that LS was associated with less blood loss, faster recovery of patients, less pain, shorter hospital stay as well as longer operation time (12-16). Some meta-analysis also demonstrated similar results (17). In terms of long-term outcomes of prospective randomized studies, only that of small samplesized studies is available. Regarding more scientifically reliable clinical studies, multi-institutional prospective randomized phase-III studies are ongoing in East Asian countries (Table 1). In China, CLASS-01 (NCT01609309) has been conducted and patients' enrollment has been completed (18). Its short-term outcomes were already published, demonstrating no difference between laparoscopic and open group $(15.2 \%$ vs. $12.9 \%)$ in morbidity rate. The primary endpoint of this study is 3 -year disease free survival, thus final outcomes is expected to be analyzed in 2018. In Korea, KLASS-02 (NCT01456598) has already completed patients' enrollment. Its short-term outcomes were disclosed in ASCO 2016 meeting (19), which demonstrated less overall complication rate $(16.4 \%$ 
vs. $24.3 \%$ ), less use of analgesics, and faster recovery in LS group. Its final analyses will be done also in 2018. In Japan, JLSSG 0901 (UMIN000003420) has completed patients' recruitment in August 2016 (20,21). The result of its phaseII part has been published, demonstrating feasible outcomes of LS, with the incidence of pancreatic fistula or anastomotic leakage as $4.7 \%$ and that of the grade 3 or higher morbidity as $5.8 \%$. Anyway, the long-term outcomes of these three clinical trials are being waited to obtain clear insight to answer our clinical questions. Although it is a randomized phase-II trial, the long-term out of COACT 1001 (NCT01088204) in Korea has been published in 2017 (22). The primary endpoint of this trial was noncompliance rate of the lymph node dissection to evaluate feasibility. The overall noncompliance rate of the lymph node dissection was not different between two groups, but in the subgroup analysis, that of LS was significantly higher for cStageIII disease $(52.0 \%$ vs. $25.0 \%)$. Interestingly, they measured several parameters for surgical stress, such as IN-6, IL-10 or TNF- $\alpha$ during the perioperative period, but there was no significant difference between two arms. Similarly, not prospective studies, but some well-designed retrospective studies using large-sized cohort have been published, in which confounding selection bias or background difference between two interventions have been balanced by method of propensity score matching. One such Chinese study demonstrated comparable oncological outcomes in two arms, but in far-advanced stage ( $\mathrm{T} 4 \mathrm{aN} 3 \mathrm{~b})$ survival rate in LS was inferior to open surgery (23). One Korean study also conducted a similar case-control study, showing comparable outcomes to open surgery; however the cohort of this study included small number of StageIII (24).

\section{Future perspective}

For reaching conclusion, we should wait for the final results of abovementioned phase-III trials. It should be noted that not only survival rate but also difference of recurrence pattern should be fully accessed to check the presence of specific tendency in recurrences after LS. Meanwhile, it is also a fact that surgical techniques are going to be refined year by year. Super high definition or three-dimensional images may help surgeons overcome limitation or shorted their learning curve. Robotic surgical instruments seem to have potential to cover disadvantages of LS, such as insufficiency of forceps' degree of freedom or surgeons' physiological tremor at the tip of device (25). It is suggested by experts that the use of a surgical robot may be beneficial for more complicated procedures, including more advanced cancer disease. However, presently, scientific evidences are lacking, and there are still several issues to be solved, such as longer operation time or expensive cost; therefore emerge of the next generation surgical robot is expected. Recently, some researchers emphasized that early initiation of adjuvant chemotherapy is correlated with better prognosis in pStageII/III gastric cancer patients (26). It is also worth evaluating whether faster patients' recovery obtained by LS can realize faster initiation of subsequent treatment for the patients (27).

\section{Conclusions}

There are still several unsolved issues regarding expanding indication of laparoscopic gastrectomy to more AGC disease. We should evaluate its validity from many aspects using the data obtained from well-designed clinical studies. At the same time, we should pay attention to ongoing technological development regarding minimal invasive surgery.

\section{Acknowledgements}

None.

\section{Footnote}

Conflict of Interest: The authors have no conflicts of interest to declare.

\section{References}

1. Japanese Gastric Cancer Association. Japanese Gastric Cancer treatment guidelines 2014 (ver.4). Gastric Cancer 2017;20:1-19.

2. Fujita J, Kurokawa Y, Sugimoto T, et al. Survival benefit of bursectomy in patients with resectable gastric cancer: interim analysis results of a randomized controlled trial. Gastric Cancer 2012;15:42-8.

3. Terashima M, Doki Y, Kurokawa Y, et al. Primary results of a phase III trial to evaluate bursectomy for patients with subserosal/serosal gastric cancer (JCOG1001). J Clin Oncol 2017;35:abstract 5.

4. Etoh T, Sasako M, Ishikawa K, et al. Extranodal metastasis is asn indicator of poor prognosis in patients with gastric carcinoma. Br J Surg 2006;93:369-73. 
5. Lee IS, Park YS, Ryu MH, et al. Impact of extranodal extension of prognosis in lymph node-positive gastric cancer. Br J Surg 2014;101:1576-84.

6. Sano T, Sasako M, Mizusawa J, et al. Randomized Controlled Trial to Evaluate Splenectomy in Total Gastrectomy for Proximal Gastric Carcinoma. Ann Surg 2017;265:277-83.

7. Kinoshita T, Shibasaki H, Enomoto N, et al. Laparoscopic splenic hilar lymph node dissection for proximal gastric cancer using integrated threedimensional anatomic simulation software. Surg Endosc 2016;30:2613-9.

8. Kinoshita T, Gotohda N, Kato Y, et al. Laparoscopic transhiatal resection for Siewert type II adenocarcinoma of the esophagogastric junction: operative technique and initial results. Surg Laparosc Endosc Percutan Tech 2012;22:e199-203.

9. Huang CM, Lv CB, Lin JX, et al. Laparoscopic-assisted versus open total gastrectomy for Siewert type II and III esophagogastric junction carcinoma: a propensity scorematched case-control study. Surg Endosc 2016. [Epub ahead of print].

10. Takiguchi S, Miyazaki Y, Shinno N, et al. Laparoscopic mediastinal dissection via an open left diaphragm approach for advanced Siewert type II adenocarcinoma. Surg Today 2016;46:129-34.

11. Inaba K, Satoh S, Ishida Y, et al. Overlap method: novel intracorporeal esophagojejunostomy after laparoscopic total gastrectomy. J Am Coll Surg 2010;211:e25-9.

12. Kelly KJ, Selby L, Chou JF, et al. Laparoscopic versus open gastrectomy for gastric adenocarcinoma in the West: a case-control study. Ann Surg Oncol 2015;22:3590-6.

13. Greenleaf EK, Sun SX, Hollenbeak CS, et al. Minimally invasive surgery for gastric cancer: the American experience. Gastric Cancer 2017;20:368-78.

14. Shinohara T, Satoh S, Kanaya S, et al. Laparoscopic versus open D2 gastrectomy for advanced gastric cancer: a retrospective cohort study. Surg Endosc 2013;27:286-94.

15. Park DJ, Han SU, Hyung WJ, et al. Long-term outcomes after laparoscopy-assisted gastrectomy for advanced gastric cancer: large-scale multicenter retrospective study. Surg Endosc 2012;26:1548-53.

16. Lee JH, Lee CM, Son SY, et al. Laparoscopic versus open gastrectomy for gastric cancer: Long-term oncologic results. Surgery 2014;155:154-64.

17. Wei HB, Wei B, Qi CL, et al. Laparoscopic versus open gastrectomy with D2 lymph node dissection for gastric cancer: a meta-analysis. Surg Laparosc Endosc Percutan
Tech 2011;21:383-90.

18. Hu Y, Huang C, Sun Y, et al. Morbidity and mortality of laparoscopic versus open D2 distal gastrectomy for advanced gastric cancer: a randomized controlled trial. J Clin Oncol 2016;34:1350-7.

19. Lee HJ, Hyung WJ, Yang HK, et al. Morbidity of laparoscopic distal gastrectomy with D2 lymphadenectomy compared with open distal gastrectomy for locally advanced gastric cancer: Short term outcomes from multicenter randomized controlled trial (KLASS-02). J Clin Oncol 2016;34:abstr 4062.

20. Inaki N, Etoh T, Ohyama T, et al. A Multi-institutional, Prospective, Phase II Feasibility Study of LaparoscopyAssisted Distal Gastrectomy with D2 Lymph Node Dissection for Locally Advanced Gastric Cancer (JLSSG0901). World J Surg 2015;39:2734-41.

21. Etoh T, Shiroshita H, Shiraishi N, et al. Ongoing clinical studies of minimally invasive surgery for gastric cancer in Japan. Transl Gastroenterol Hepatol 2016;1:31.

22. Park YK, Yoon HM, Kim YW, et al. Laparoscopy-Assisted versus Open D2 Distal Gastrectomy for Advanced Gastric Cancer: Results from a Randomized Phase II Multicenter Clinical Trial (COACT 1001). Ann Surg 2017. [Epub ahead of print].

23. Lin JX, Huang CM, Zheng CH, et al. Is all advanced gastric cancer suitable for laparoscopy-assisted gastrectomy with extended lymphadenectomy? A case-control study using a propensity score method. Ann Surg Oncol 2016;23:1252-60.

24. Kim HH, Han SU, Kim MC, et al. Long-term results of laparoscopic gastrectomy for gastric cancer: a large-scale case-control and case-matched Korean multicenter study. J Clin Oncol 2014;32:627-33.

25. Terashima $M$, Tokunaga $M$, Tanizawa $Y$, et al. Robotic surgery for gastric cancer. Gastric Cancer 2015;18:449-57.

26. Park HS, Jung M, Kim HS, et al. Proper timing of adjuvant chemotherapy affects survival in patients with stage 2 and 3 gastric cancer. Ann Surg Oncol 2015;22:224-31.

27. Kaito A, Kinoshita T, Shitara K, et al. Timing of initiation of adjuvant chemotherapy for gastric cancer: A casematched comparison study of laparoscopic vs. open surgery. Eur J Surg Oncol 2017;43:801-7.

doi: $10.21037 / \operatorname{tgh} .2017 .04 .05$

Cite this article as: Kinoshita T, Kaito A. Current status and future perspectives of laparoscopic radical surgery for advanced gastric cancer. Transl Gastroenterol Hepatol 2017;2:43. 